\title{
Dilepton and Trilepton Production by Antineutrinos and Neutrinos in Neon
}

\author{
BEBC WA 59 Collaboration
}

G. Gerbier ${ }^{12}$, D. Bertrand ${ }^{3 a}$, J. Guy ${ }^{11}$, P. Marage ${ }^{3 b}$, M. Aderholz ${ }^{9}$, N. Armenise ${ }^{1}$, J.H. Bartley ${ }^{14}$, J.P. Baton ${ }^{12}$, V. Brisson ${ }^{6}$, R. Belusevic ${ }^{2}$, D. Brou ${ }^{12}$, F.W. Bullock ${ }^{14}$, M. Calicchio ${ }^{1}$, E.F. Clayton ${ }^{7}$, T. Coghen ${ }^{8}$, A.M. Cooper-Sarkar ${ }^{11}$, O. Erriquez ${ }^{1}$, P.J. Fitch ${ }^{14 c}$, P.O. Hulth ${ }^{13}$, G.T. Jones ${ }^{2}$, P. Kasper ${ }^{12}$, H. Klein ${ }^{4}$, C. Kochowski ${ }^{12}$, M. Lagraa ${ }^{12}$, S. Leighton-Davis $^{14 \mathrm{c}}$, R. Middleton ${ }^{2 c}$, D.B. Miller ${ }^{7}$, M.M. Mobayyen ${ }^{7}$, D.R.O. Morrison ${ }^{4}$, M. Neveu ${ }^{12}$, S. Nuzzo ${ }^{1}$, S.O'Neale ${ }^{2}$, M.A. Parker ${ }^{4}$, P. Petiau ${ }^{6}$, J. Sacton ${ }^{3}$, R.A. Sansum ${ }^{14 c}$, N. Schmitz ${ }^{9}$,

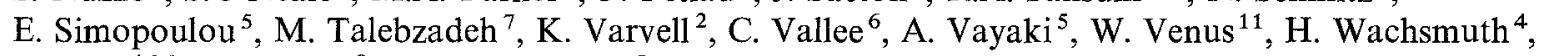
J. Wells ${ }^{10 d}$, W. Wittek ${ }^{9}$, E. Zevgolatakos ${ }^{5}$

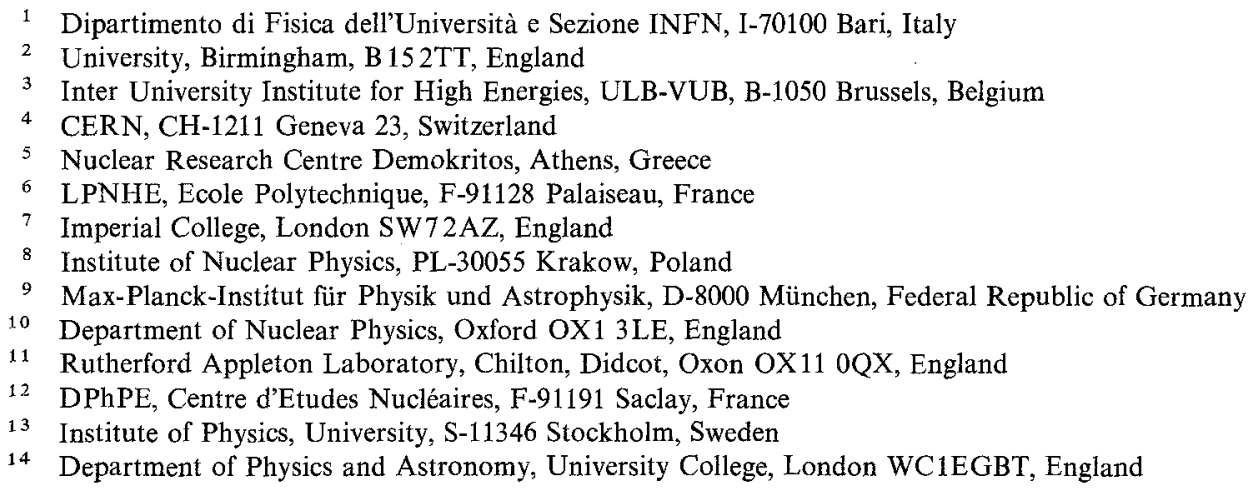

Received 29 May 1985

\begin{abstract}
A sample of over 25,000 fully measured neutrino and antineutrino charged current interactions in BEBC includes 192 dilepton candidates. The prompt signal after subtraction of background is 41 $\pm 7 \mu^{+} e^{-}, 35 \pm 7 \mu^{+} \mu^{-}$events from $\bar{v}$ interactions, and $32 \pm 7 \mu^{-} \mu^{+}$events from $v$ interactions. There are 2 trileptons, $\mu^{-} \mu^{-} e^{+}$and $\mu^{-} \mu^{-} \mu^{+}$. Results are compared with other experimental data and with the standard model. Limits to prompt like sign $\mu^{+} e^{+}$, $\mu^{+} \mu^{+}$and $\mu^{-} \mu^{-}$signals are given and compared with other experiments and with theoretical calculations.
\end{abstract}

a Chercheur qualifié FNRS, Belgique

b Chercheur sur Action de Recherche Concertée - Convention 84/89-63 - Belgique

- Supported by a SERC Studentship

d Now at Department of Natural Philosophy, University of Glasgow

\section{Introduction}

Production of dilepton events by neutrinos and antineutrinos, attributed to charmed particle production and semileptonic decay, has been studied in several experiments. Counter experiments [1] have accumulated large samples of events with two muons (dimuons or $\mu \mu$ events). Bubble chambers [2] have accumulated smaller samples of both $\mu \mu$ and $\mu e$ events which are expected to have the same origin and have provided details of the accompanying hadronic system. However, the data from antineutrino experiments in bubble chambers are still quite fragmentary, being based on the observation of small numbers of events collected in different experiments.

In the present paper, a study is presented of antineutrino induced dimuons observed in the $\mathrm{BEBC}$ film recently used for an analysis of $\mu e$ events [6], allowing a comparison of the general character- 
istics of both sets of data. A further comparison is made with the results of a similar study of dimuons produced by neutrinos. The neutrino induced $\mu e$ events were not analyzed since two bubble chamber experiments with higher statistics have already presented results $[4,5]$. Special attention has been paid to the like sign dilepton events for which the experimental situation is still controversial.

\section{Experimental Set Up}

The Big European Bubble Chamber (BEBC) was exposed to the CERN SPS two horn antineutrino (neutrino) wide band beam obtained with $400 \mathrm{GeV}$ incident protons. The horns were set to focus negative secondaries ( $\bar{v}$ run) or positive secondaries ( $v$ run) in two consecutive exposures. The resulting antineutrino (neutrino) charged current events have a mean energy of $40(55) \mathrm{GeV}$.

The bubble chamber was filled with a 75 mole percent neon-hydrogen mixture with a radiation length of $42 \mathrm{~cm}$. This liquid acts as an excellent electron identifier, while allowing complete measurement of individual secondary particles.

The External Muon Identifier (EMI) consists of two planes of wire chambers (the inner and outer planes) flanking and downstream of BEBC [7]. The inner plane has 8 overlapping chambers which are $150 \mathrm{~cm}$ from BEBC, (including $50 \mathrm{~cm}$ of magnet coils or lead). Muons then traverse between 5 and 10 interaction lengths of iron hadron absorber before reaching the outer plane, made of 47 overlapping chambers. The readout of all the chambers was triggered by any hit occurring in either the inner or outer plane. The total spill length of $4 \mathrm{~ms}$ was divided into time slots of $250 \mathrm{~ns}$. Hits occurring in two adjacent time slots were merged, leading to an effective time resolution of $500 \mathrm{~ns}$ and no dead time.

\section{Event Selection}

The BEBC pictures were double-scanned for all neutral induced interactions occurring within the visible chamber volume. In a first step, for each event located in a restricted fiducial volume of $17 \mathrm{~m}^{3}$, all charged secondaries leaving the chamber that could be of momentum greater than $3 \mathrm{GeV} / \mathrm{c}$ were measured as muon candidates. All the events containing at least one positively (in the $\bar{v}$ run) or one negatively (in the $v$ run) charged muon candidate were then fully measured. The chosen fiducial volume allows at least $50 \mathrm{~cm}$ downstream from each event vertex for detection and measurement of associated neutrals $\left(\gamma, K^{0}, \Lambda^{0}\right.$, neutrons).
The trajectories of the leaving particles were extrapolated to the inner and the outer planes of the EMI. A particle was considered as a muon if hits were found in both planes within the same time slot and at the expected positions, taking into account the error in the extrapolation due to multiple scattering and measurement errors. The backgrounds included in the muon classification are small in general, and are studied in detail for the dileptons in Sect. 5.2. Events with an identified muon of momentum over $4 \mathrm{GeV} / \mathrm{c}$ were finally accepted as charged current $(\mathrm{CC})$ events. This yielded a measured sample of $16,600 \bar{v}_{\mu}$ CC events and 3,300 $v_{\mu}$ CC from the $\bar{v}$ run, and $9,650 v_{\mu} \mathrm{CC}$ and essentially no $\bar{v}_{\mu}$ from the $v$ run.

In the $\bar{v}$ run, the $\mu^{+} e$ events were selected by reexamining the identified $\bar{v}_{\mu}$ induced CC events to see if any tracks gave characteristic electron signatures. An event containing an electron or positron candidate which could not be paired with one of opposite charge (to form a Dalitz pair or close gamma conversion), showed at least two electron signatures and had a momentum greater than $300 \mathrm{MeV} / \mathrm{c}$ was considered as a $\mu^{+} e$ candidate. As a results of this search, $55 \mu^{+} e^{-}$and $4 \mu^{+} e^{+}$candidates have been detected in a subsample of 15,370 antineutrino charged current interactions. Requiring the electron (positron) momentum to be above $800 \mathrm{MeV} / \mathrm{c}$ reduces these numbers to $47 \mu^{+} e^{-}$and $3 \mu^{+} e^{+}$respectively. The $v_{e}$ and $\bar{v}_{e}$ events were not selected; however any that also had a $\mu^{+}$are included.

In both runs, if an event contained one identified muon of momentum greater than $4 \mathrm{GeV} / \mathrm{c}$ then any additional muon candidate within the same time slot had only to be above $3 \mathrm{GeV} / \mathrm{c}$ to be accepted and could have just a single outer plane EMI hit if its trajectory missed the inner EMI plane. In the $\bar{v}$ run $64 \mu^{+} \mu^{-}, 7 \mu^{+} \mu^{+}$and $5 \mu^{-} \mu^{-}$events were so selected. In the $v$ run there were $46 \mu^{-} \mu^{+}, 9 \mu^{-} \mu^{-}$, $1 \mu^{-} \mu^{-} \mu^{+}$and $1 \mu^{-} \mu^{-} e^{+}$event.

All multilepton events were carefully re-checked to ensure that all hadrons were measured as well as possible, and that all associated neutrals had been found.

\section{Separation of Anti-Neutrino From Neutrino Induced Dileptons}

Dilepton events collected in this experiment can be produced by any type of neutrino present in the beam: $v_{\mu}, \bar{v}_{\mu}, v_{e}$ or $\bar{v}_{e}$. The primary lepton associated with the incident neutrino was distinguished from the secondary lepton by comparing their longitudinal and transverse momenta with respect to the beam direction, $P_{l}$ and $P_{t}$. In general both the $P_{l}$ and 

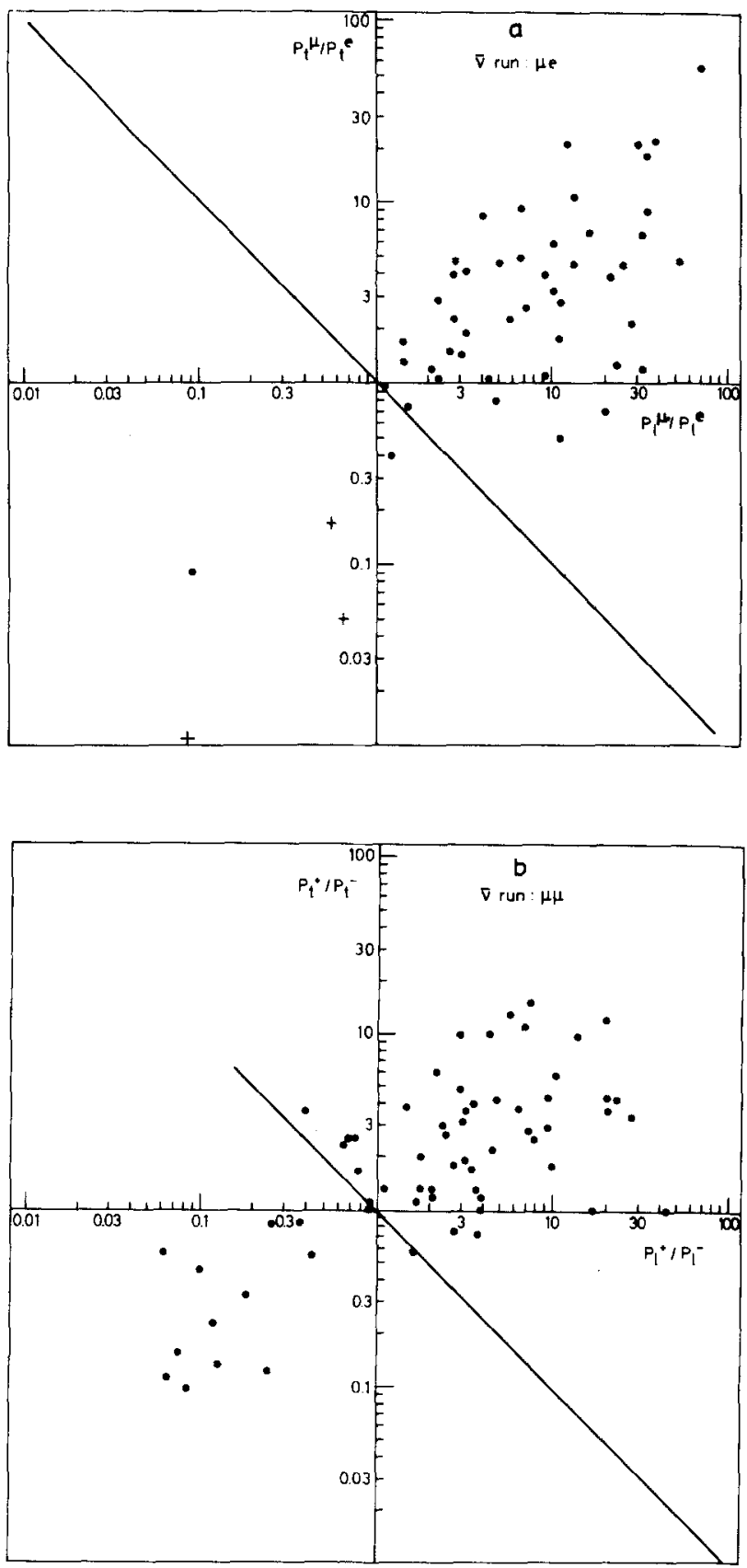

$P_{t}$ of the primary lepton will be larger than those of the secondary lepton. This distinction was made without reference to the hadron shower direction. Figure 1 shows the ratio of the longitudinal momenta of the two leptons versus the ratio of their transverse momenta. For the $\bar{v}$ run the $49 \mu^{+} \mu^{-}$events above the diagonal line on Fig. $1 \mathrm{~b}$ were attributed to $\vec{v}_{\mu}$ interactions. The $15 \mu^{-} \mu^{+}$events below the line were attributed to $v_{u}$ interactions. The $\mu^{+} e$ events in the $\bar{v}$ run were treated in the same way. Thus the $45 \mu^{+} e^{-}$and $0 \mu^{+} e^{+}$events above the diagonal line in Fig. 1a were attributed to $\bar{v}_{e}$ interactions whereas,

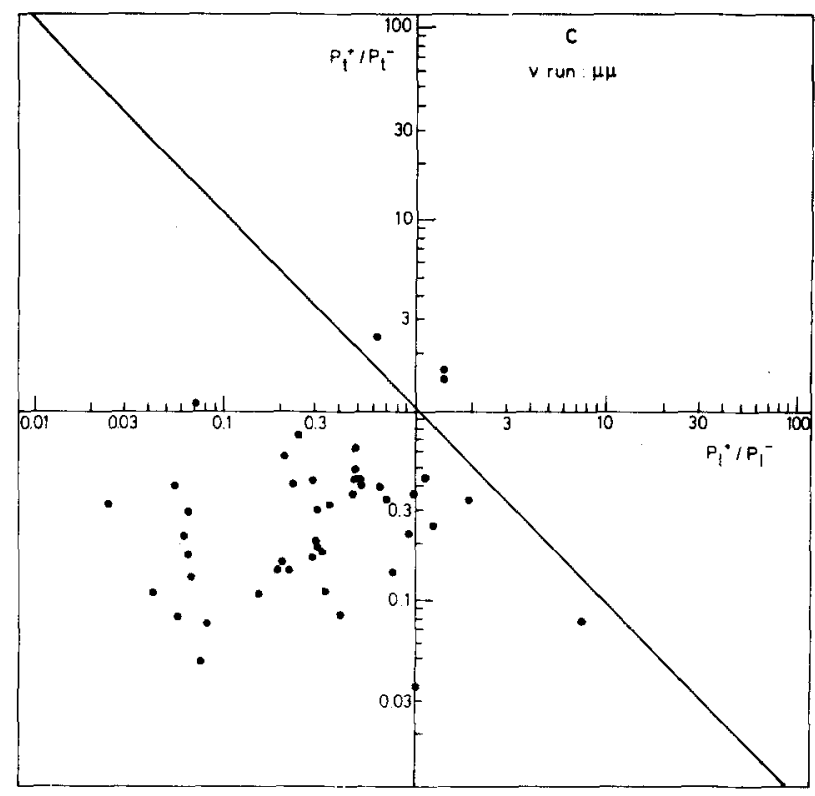

Fig. $1 \mathrm{a}-\mathrm{c}$. Separation of $\vec{v}$ and $v$ interactions. For dimuons the scatter plot shows the ratio of the transverse momenta of the $\mu^{+}$ and the $\mu^{-}$(with respect to the neutrino direction) versus the ratio of their longitudinal momenta; a) $\bar{v}$ run; $\cdot \mu^{+} e^{-},+\mu^{+} e^{+}$; b $\bar{v}$ run; $\mu^{+} \mu^{-} ; \mathbf{c} v$ run $; \mu^{+} \mu^{-}$

below this line $3 e^{+} \mu^{+}$events where attributed to $\bar{v}_{e}$ interactions and $2 e^{-} \mu^{+}$to $v_{e}$ interactions. It should be noticed that a low energy muon candidate in a $v_{e}$ or $\bar{v}_{e}$ event has a much greater chance of being due to background hits in the EMI than the second muon in a dimuon event since there is no primary muon to select the correct EMI time slot.

A Monte Carlo simulation indicates that for $\bar{v}$ interactions about $10 \%$ of genuine dimuons coming from charm decay are misclassified by application of the above procedure; this fraction is $\sim 5 \%$ both for $\bar{y}$-induced $\mu e$ events and for $\nu$-induced dimuon 
events. For the $v$ run, $43 \mu^{-} \mu^{+}$events lie below the diagonal line in Fig. $1 \mathrm{c}$ and only 3 events are above this line, in agreement with this prediction.

\section{Backgrounds}

\subsection{Backgrounds to Prompt $\mu^{+}$e Events}

It has been shown [6] that the major sources of background which simulate genuine $\mu^{+} e$ events are asymmetric Dalitz pairs, and Compton electrons or asymmetric $e^{+} e^{-}$pairs appearing to be attached to the primary vertex because of the limited bubble chamber resolution. These sources mainly contribute at low electron momenta: between 300 and $800 \mathrm{Me}-$ $\mathrm{V} / \mathrm{c}$, these backgrounds contribute $(4.7 \pm 0.7) \mu^{+} e^{--}$ events and $(0.7 \pm 0.1) \mu^{+} e^{+}$events, whilst for momenta above $800 \mathrm{MeV} / \mathrm{c}$ these numbers are reduced to $(2.0 \pm 0.4)$ and $(0.2 \pm 0.1)$ events respectively. As in many other experiments, an electron momentum cut-off of $800 \mathrm{MeV} / \mathrm{c}$ has been applied to the $\mu^{+} e^{-}$events. The one observed $\mu^{+} e^{+}$event with $e^{+}$ momentum between 300 and $800 \mathrm{MeV} / \mathrm{c}$ is discussed in Sect. 7.4.

The accepted sample of $45 \mu^{+} e^{-}$events induced by $\bar{v}_{\mu}$ includes a total background of $(3.9 \pm 1.6)$ events. A full discussion of the backgrounds is given in [6].

\subsection{Background to Prompt Dimuon Events}

The major background to dimuon events is due to decay muons from $\pi$ and $K$ mesons in the hadronic shower in normal charged current events $\star$ Its contribution of the event vertices in the chamber volsimulation using as input the measured momenta and angles of the hadrons produced in the present sample of charged current interactions and the distribution of the event vertices in the chamber volume. The hadrons, assumed to be $\pi$ or $K$ mesons in the proportion of 8 to 1 , which left the chamber without visible interaction were followed step by step and allowed to decay; the decay muons were then followed to the EMI. This simulation took into account the magnetic field and the effects of energy losses and multiple scattering in the media traversed. It showed that these decay muons tend to give poorer associated extrapolations in the EMI than genuine prompt muons, because the geometrical track parameters measured in the chamber and extrapolat-

\footnotetext{
* All muons with momenta $>3 \mathrm{GeV} / \mathrm{c}$ from $\pi$ decay and about $\frac{1}{3}$ of those from $K$ decay are emitted at an angle less than $50 \mathrm{mrad}$, corresponding to the empirical limit of visibility in the chamber for this experiment
}

Table 1. Numbers of observed dimuon events and expected background events $\left(P_{\mu_{1}}>4 \mathrm{GeV} / \mathrm{c}, P_{\mu_{2}}>3 \mathrm{GeV} / \mathrm{c}\right)$

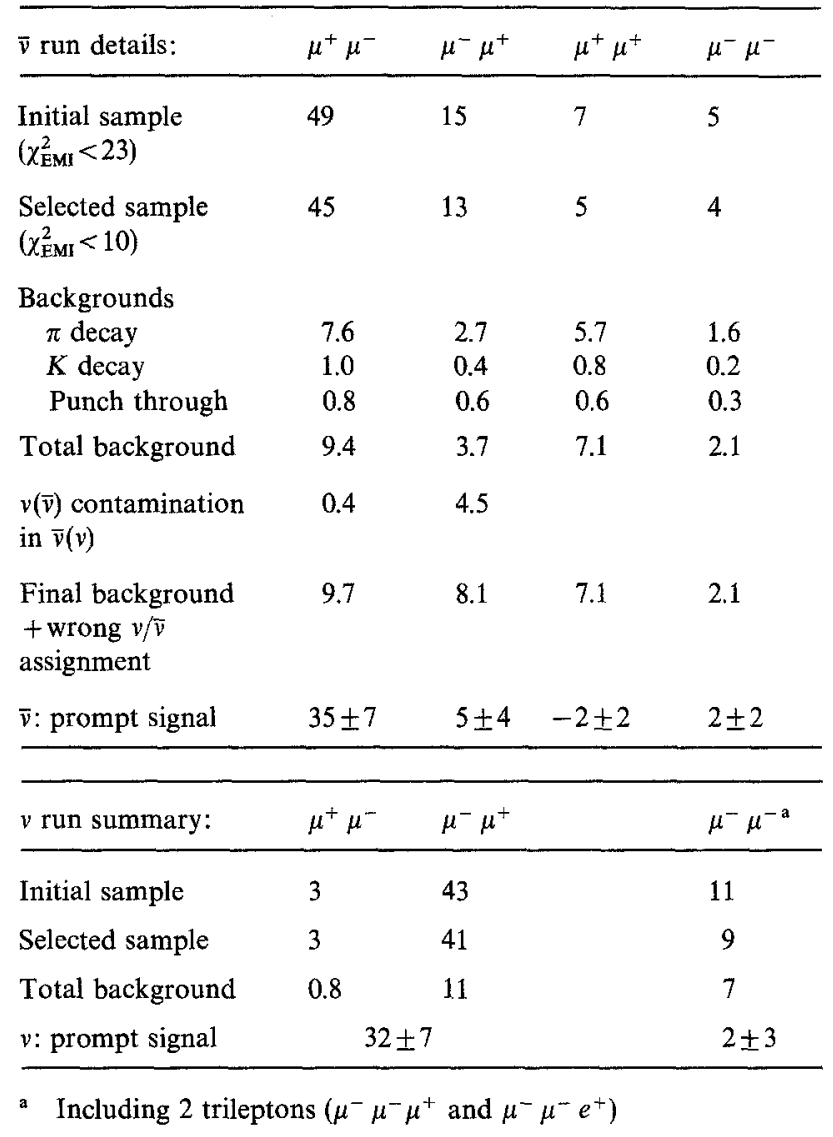

ed to the EMI are often closer to those of the parent hadrons. For the selection of dimuon events, stronger cuts $\left(\chi_{\text {EMI }}^{2}\right.$ less than 10 for 2 degrees of freedom) were therefore applied to the association probability of extrapolated secondary tracks with EMI hits than in the case of normal primary muons from chargedcurrent events; this reduced the background by a larger factor than the prompt dimuon signal.

The results of this background estimation are summarized in Table 1; its momentum dependence is given in Table 2. The systematic errors on the hadron decay backgrounds are estimated to be of the order of $\pm 15 \%$.

There is also a $2 \%$ background due to hadron "punch through" caused by intense hadron showers from a charged current event reaching the second plane of the EMI and simulating a muon [8]. Random association of an extrapolated track and background hits in the EMI time slot defined by the primary muon has been determined to be negligible.

The total background is $(9.7 \pm 1.5)$ events among 45 accepted $\mu^{+} \mu^{-}$events in the $\bar{v}$ run, and it is (11 \pm 2 ) among the 41 accepted $\mu^{-} \mu^{+}$events in the $v$ run. In both runs, the number of like sign events is 
Table 2. $\bar{v}$ run: numbers of $\mu^{+} \mu^{-}$events, backgrounds and efficiencies; shown as a function of the $\mu^{-}$momentum

\begin{tabular}{lccccc}
\hline$\mu^{-}$momentum $(\mathrm{GeV} / \mathrm{c})$ & $3-5$ & $5-7$ & $7-10$ & $>10$ & Total \\
\hline $\begin{array}{l}\text { Accepted } \mu^{+} \mu^{-} \\
\text {Background }+\nu\end{array}$ & 16 & 10 & 8 & 11 & 45 \\
$\quad$ contamination & 4.1 & 2.5 & 1.7 & 1.5 & 9.7 \\
Prompt $\mu^{-}$ & 11.9 & 7.5 & 6.3 & 9.5 & 35.3 \\
& & & & & \\
Efficiencies (in \%) & & & & & \\
EMI acceptance & 85 & 93 & 98 & 99 & 93 \\
$\chi_{\text {EMI }}^{2}<10$ & 95 & 97 & 96 & 98 & 96 \\
Remaining losses of & 87 & 90 & 90 & 94 & 90 \\
$\quad \mu^{-}$(see text) & 98 & 95 & 93 & 75 & 90 \\
Kept as $\bar{v}$ & 17 & 10 & 8 & 14 & 49 \\
Corrected $\mu^{+} \mu^{-}$ & & & & & \\
\hline
\end{tabular}

consistent with the expected background (see Table 1).

\section{Losses of Dilepton Events}

In addition to the misidentification due to the $v / \tilde{v}$ separation procedure discussed in Sect. 4, various losses of dilepton events are expected.

\subsection{Losses of $\mu^{+}$e Events}

The electron scan and detection efficiencies have been studied in detail in [6]. The most significant loss of $\mu^{+} e$ events with $p_{e}>0.8 \mathrm{GeV} / \mathrm{c}$ is due to the limited size of the bubble chamber and the requirement of two signatures for the identification of the electron; this identification efficiency is estimated to be $(90 \pm 3) \%$, independent of the electron momentum.

\subsection{Losses of Dimuon Events}

Muons with momentum below $3 \mathrm{GeV} / \mathrm{c}$ seldom reach the EMI, because of the hadron absorber, the magnetic field between the bubble chamber and the EMI, and the limited EMI angular coverage. Above $3 \mathrm{GeV} / \mathrm{c}$, the effects of the EMI acceptance and of the $\chi_{\text {EMI }}^{2}$ cut at 10 are shown in Table 2 for the $\bar{v}$ run as a function of secondary momentum.

The remaining losses of muons above $3 \mathrm{GeV} / \mathrm{c}$ were evaluated using three different methods, all based on statistical evaluations of the number of unidentified charged current events remaining among the events classified as neutral current or neutral hadron induced interactions. These methods [8] are based respectively on

(i) comparing the track length distributions of a) identified muons, b) hadrons in charged current events and c) particles leaving the chamber from events with no identified muon,

(ii) comparing a kinematic selection of charged current events with the EMI selection,

(iii) comparing the measured number of events with no identified muon with the number expected on the basis of different contributing channels.

These separate estimates of the remaining losses of the secondary muon are consistent and are combined to give $(10 \pm 3) \%$ in Table 2 .

\section{Results}

\subsection{Cross Sections}

The number of prompt opposite sign dilepton events per $\mathrm{CC}$ event, with the listed cuts on lepton momenta and corrected for backgrounds and losses, are shown in Table 3. This table also gives the rates corrected for the cuts on the lepton momenta, according to the model presented in Sect. 7.2 for production and semi-leptonic decay of charmed particles.

Table 3 also allows a direct comparison of $\mu^{+} e^{-}$ and $\mu^{+} \mu^{-}$production rates in the $\bar{v}$ run, with the same cuts $\left(p_{\mu^{+}}>4 \mathrm{GeV} / \mathrm{c}, p_{\mu^{-}}\right.$and $\left.p_{e^{-}}>3 \mathrm{GeV} / \mathrm{c}\right)$, without charm model dependent corrections; although the $\mu^{+} \mu^{-}$rate is higher than the $\mu^{+} e^{-}$rate, they are compatible within statistics.

\subsection{Comparison with a Charm Production Model}

The 3 samples of dilepton events were compared with the predictions for the production and semileptonic decay of charmed $D$ mesons based on the Lund Monte Carlo model, with the parametrization of Field and Feynman [9]: $D^{-}$and $\bar{D}^{0}$ are produced

Table 3. Number of opposite sign dilepton events $\left(\times 10^{4}\right)$ per charged current event, corrected for backgrounds and losses

\begin{tabular}{|c|c|c|c|}
\hline $\begin{array}{l}\text { Rates } \times 10^{4}, \\
\text { cuts in } \mathrm{GeV} / \mathrm{c}\end{array}$ & $\bar{v}: \mu^{+} e^{--}$ & $\bar{v}: \mu^{+} \mu^{-}$ & $v: \mu^{-} \mu^{+}$ \\
\hline$p_{\mu_{1}}>4, p_{1_{2}}>3$ & $18 \pm 4$ & $29 \pm 6$ & $40 \pm 9$ \\
\hline$p_{\mu_{1}}>4, p_{1_{2}}>0.8$ & $31 \pm 5$ & $\pm 10^{a}$ & $\pm 15^{a}$ \\
\hline$p_{\mu_{1}}>0, p_{1_{2}}>0$ & $43 \pm 7^{a}$ & $70 \pm 18^{\mathrm{a}}$ & $75 \pm 17^{a}$ \\
\hline $\begin{array}{l}V^{0} \text { rate per prompt } \\
\text { dilepton event }\end{array}$ & $1.3 \pm 0.3$ & $0.45 \pm 0.20$ & $1.0 \pm 0.3$ \\
\hline $\begin{array}{l}\text { World } V^{0} \text { rates } \\
\text { including these }\end{array}$ & $1.5 \pm 0.4$ & $0.6 \pm 0.2$ & $0.7 \pm 0.2 \mu^{-} \mu^{+}$ \\
\hline data $[8]$ & & & $0.93 \pm 0.14 \mu^{-} e^{+}$ \\
\hline
\end{tabular}

a Opposite sign dilepton rates corrected for lepton momentum cuts 

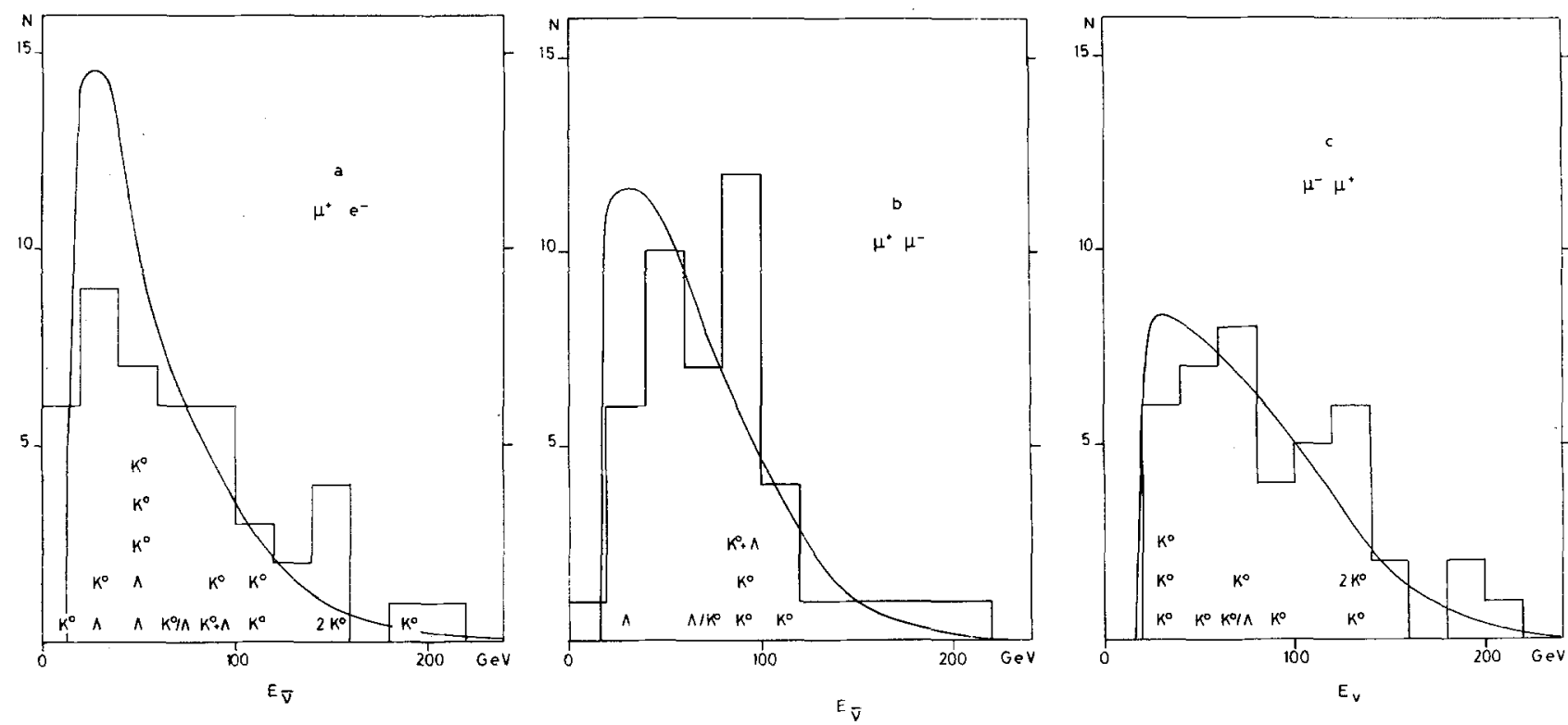

Fig. 2a-c. Distribution of the (anti)neutrino energy for: a $\mu^{+} e^{-}$events ( $\bar{v}$ run); b $\mu^{+} \mu^{-}$events ( $\bar{v}$ run); c $\mu^{-} \mu^{+}$events (v run). The events containing neutral strange particles are indicated. The curve represents the prediction of the Monte-Carlo simulation of charm production

by $\bar{v}$ interactions on $\bar{s}$ quarks of the strange sea, whereas $D^{+}$and $D^{0}$ are produced by $v$ interactions on valence $d$ quarks and on sea $s$ quarks. Appropriate fractions of background events were included in the Monte-Carlo, and losses of prompt dilepton events were accounted for, according to the above discussion. The calculated quantities were corrected for undetected neutral secondaries in the same way as the experimental quantities. Within the quite large statistical uncertainties, the Monte-Carlo predictions appear reasonably compatible with the data, as displayed in Figs. 2 to 7.

In the $\bar{v}$ run, if the Bjorken $x$ distribution of the strange sea is parameterized as $d N / d x \sim(1-x)^{\gamma}$ then $\gamma=\left(8.1_{-1.6}^{+2.6}\right)$ for the $\mu^{+} \mu^{-}$events (with a mean $Q^{2}$ of $8.6 \mathrm{GeV}^{2}$ ) and $\gamma=\left(6.1_{-0.9}^{+1.1}\right)$ for the $\mu^{+} e^{-}$. events (with a mean $Q^{2}$ of $8.7 \mathrm{GeV}^{2}$ ) - after allowing for background and smearing. The wider $x$ distribution in the case of $\nu$ induced $\mu^{-} \mu^{+}$events (Fig. 4c) is expected from the additional valence $d$ quark contribution to the charm production.

In the case of $\bar{v}$ interactions, the distribution of the invariant mass of the hadron system, $W$, peaks at about $6 \mathrm{GeV}$ for both the data and the Monte-Carlo (see Figs. $3 \mathrm{a}, \mathrm{b}$ ). The accumulation of events in this mass region - where the experimental resolution in $W$ is about $0.8 \mathrm{GeV}$ - has previously led to speculations on quasi-elastic beauty baryon production $[3,10]$. One would expect larger values of the transverse momentum of the $e^{-}$or $\mu^{-}$out of the $(\bar{v}$, lepton) plane for events with $B$ decay: $e^{-}$and $\mu^{-}$ from events with $5<W<7 \mathrm{GeV}$ are labelled in Figs.
$7 \mathrm{a}, \mathrm{b}^{\star}$. Moreover, a calculation for $B$ production by $\bar{v}_{\mu}$, based on a model for $A_{c}^{+}$quasi elastic production by $v_{\mu}[11]$ and assuming $\left|U_{u b}\right|=0.006$ [12], suggests a contribution of less than 1 semileptonic $B$ decay in the $\bar{v}$ data.

\subsection{Associated Strange Particle Production}

In antineutrino reactions, the dominant charm production on a sea $\bar{s}$ quark implies the presence of two strange particles in the final state; in neutrino induced charm production, due to the additional contribution of interactions on $d$ quarks, one expects one or two strange particles in the final state. The $45 \mu^{+} e^{-}$events have 17 associated $V^{0}$ decays, and the $45 \mu^{+} \mu^{-}$events have $7 V^{0}$ ( $\bar{v}$ run); the $41 \mu^{-} \mu^{+}$ events in the $v$ run have 10 associated $V^{0}$. The $V^{0}$ production rate is about 0.11 to $0.14(0.12$ to 0.15$)$ for ordinary charged-current $\vec{v}(v)$ events in the same $W$ range.

The $V^{0}$ rates in the prompt dilepton events, corrected for losses due to the finite length of their potential path in the liquid, decays close to the interaction vertex, interactions before decay and unseen decay modes are summarized in Table 3. Also shown are the world data $V^{0}$ rates corrected for background in a uniform way as discussed in [8].

\footnotetext{
$\star$ In the $\bar{v}$ run there are 15 events with $p_{l_{2}}^{T}$ over $0.5 \mathrm{GeV} / \mathrm{c} .8$ of these have $W$ between 5 and $7 \mathrm{GeV}$, when $(5 \pm 2)$ would be expected on the basis of the $\bar{v}$ events with $p_{l_{2}}^{T}$ below $0.5 \mathrm{GeV} / \mathrm{c}$ and the $v$ events
} 

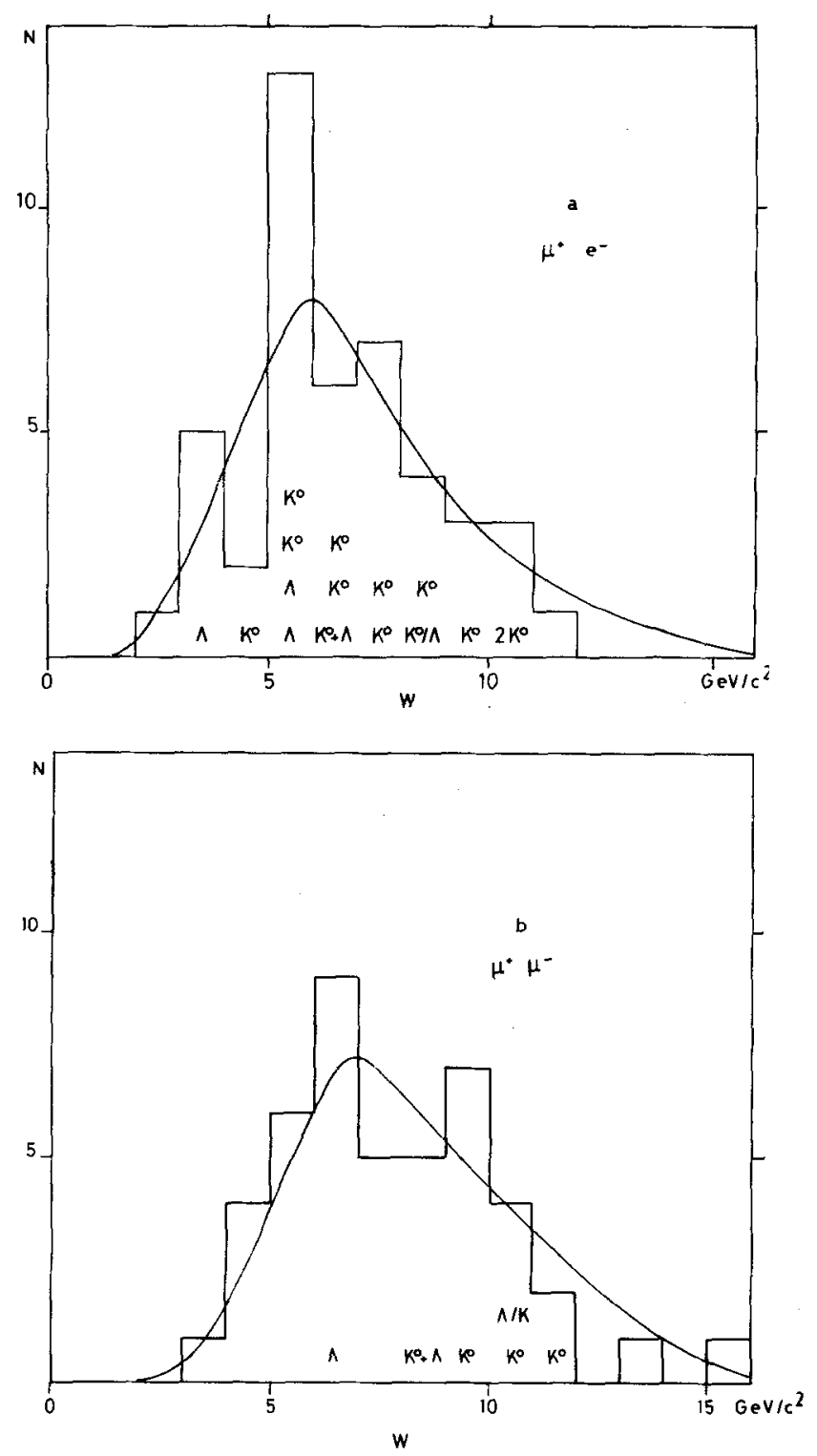

The events with a $V^{0}$ are shown in Figs. 2 to 7. Further $V^{0}$ distributions are shown in Figs. 8 and 9. Five of the $6 \Lambda^{\circ}$ hyperons have momenta below $1 \mathrm{GeV} / \mathrm{c}$, and all are produced backward in the hadronic centre of mass $\left(x_{F}<0\right)$, which is consistent with $A^{0}$ hyperon production from the fragmentation of the target remnant. $K^{0}$ mesons are produced either forward, mostly from the decay of charmed particles, or backward, mostly from the fragmentation of the remaining $s$ quark. Similar conclusions can also be inferred from the invariant mass plots of the $V^{0}$ and the second lepton.

\subsection{Same Sign Dilepton Events}

There are $3 e^{+} \mu^{+}$candidates with $e^{+}$momenta above $0.8 \mathrm{GeV} / \mathrm{c}$ in the $\bar{v}$ run which are all attributed

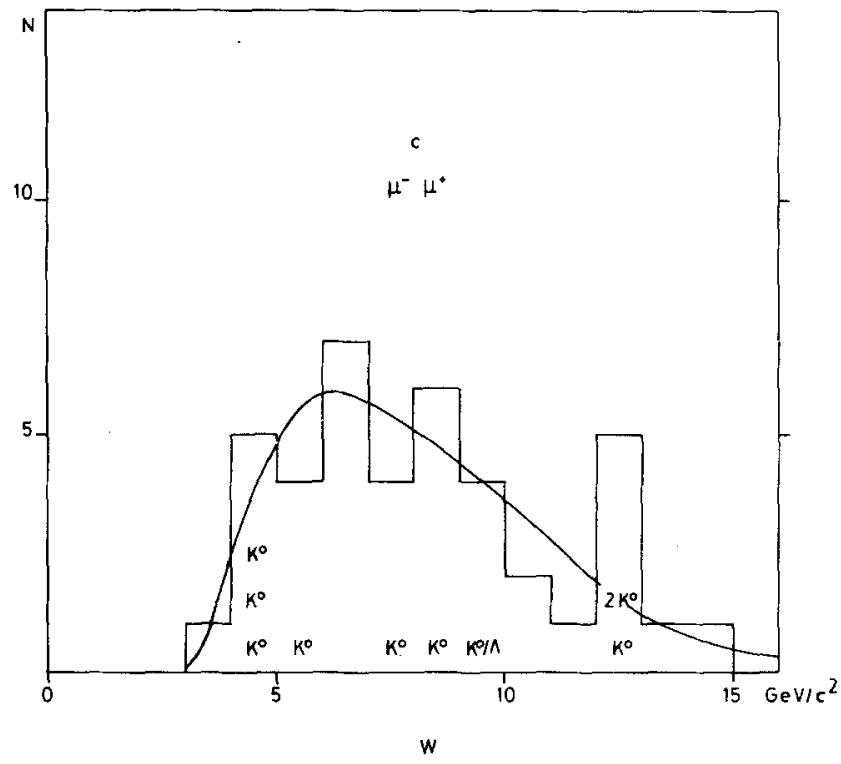

Fig. 3a-c. Distribution of the hadronic invariant mass, $W$, as for Fig. 2

to $\bar{v}_{e}$ interactions (see Sect. 4 and [6]); this is confirmed by the fact that in these events the $\mu^{+}$lies nearer than the $e^{+}$to the direction of the hadron shower. Furthermore all 3 muons have $\chi_{\mathrm{EMI}}^{2}$ greater than 10. There are no $V^{0}$ associated with these $3 e^{+} \mu^{+}$candidates.

Taking into account the losses and efficiencies we find upper limits (at 90\% confidence level) of 2 $\times 10^{-4}$ for the ratio $\left(\mu^{+} e^{+}\right.$events $) /\left(\right.$all $\mu^{+}$events) and $6 \%$ for $\left(\mu^{+} e^{+}\right.$events $) /\left(\mu^{+} e^{-}\right.$events $)$, as listed in Table 4.

There is one further $\mu^{+} e^{+}$candidate with low $e^{+}$ momentum: $(303 \pm 40) \mathrm{MeV} / \mathrm{c}$, below the $800 \mathrm{MeV} / \mathrm{c}$ momentum cut. This event has an associated decay of a $0.9 \mathrm{GeV} / \mathrm{c} \mathrm{K}^{0}$ meson. However the background due to asymmetric pairs is $(0.7 \pm 0.1)$ events for positron momentum between 0.3 and $0.8 \mathrm{GeV} / \mathrm{c}$, while a 

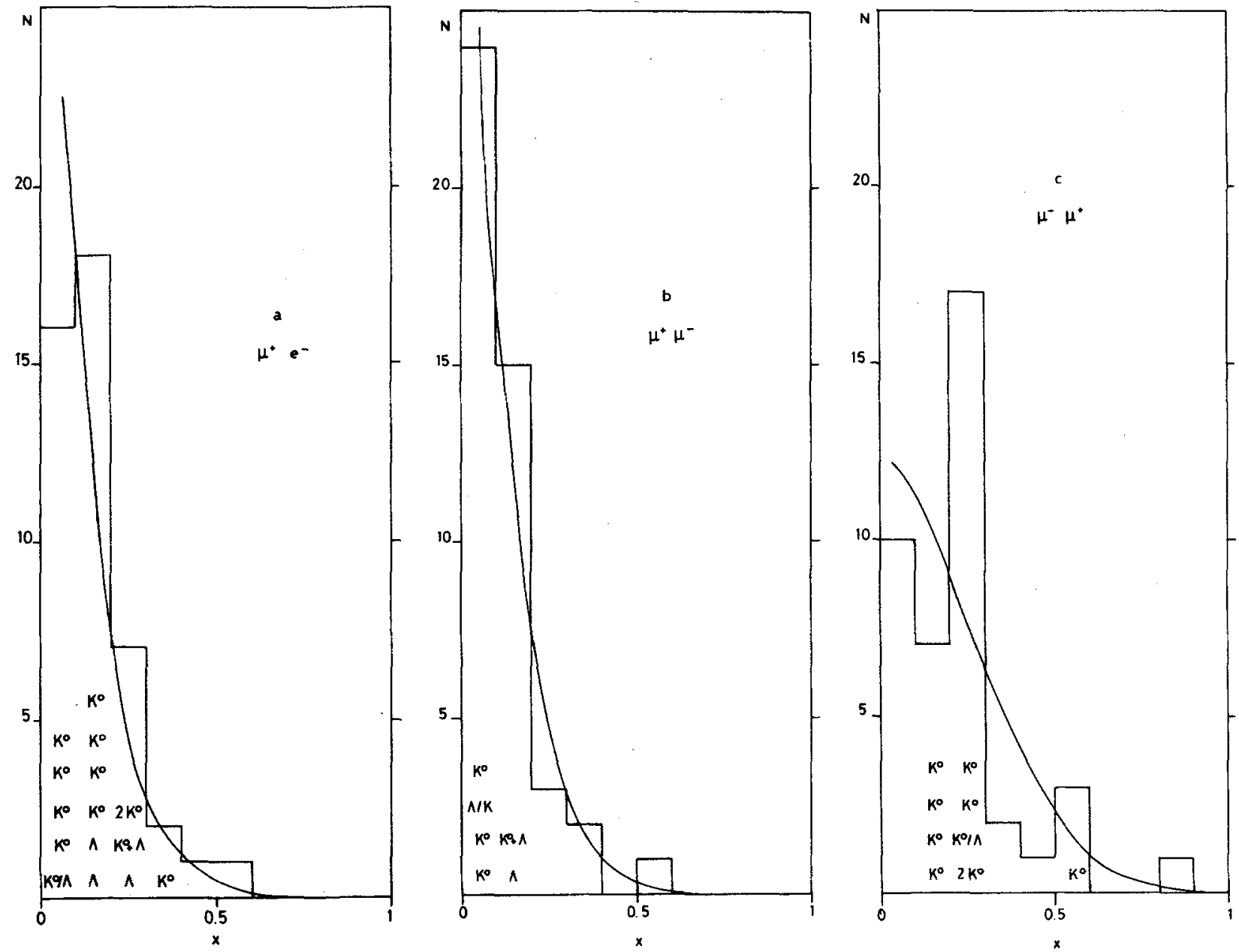

Fig. 4a-c. Distribution of the Bjorken $x$ variable, as for Fig. 2
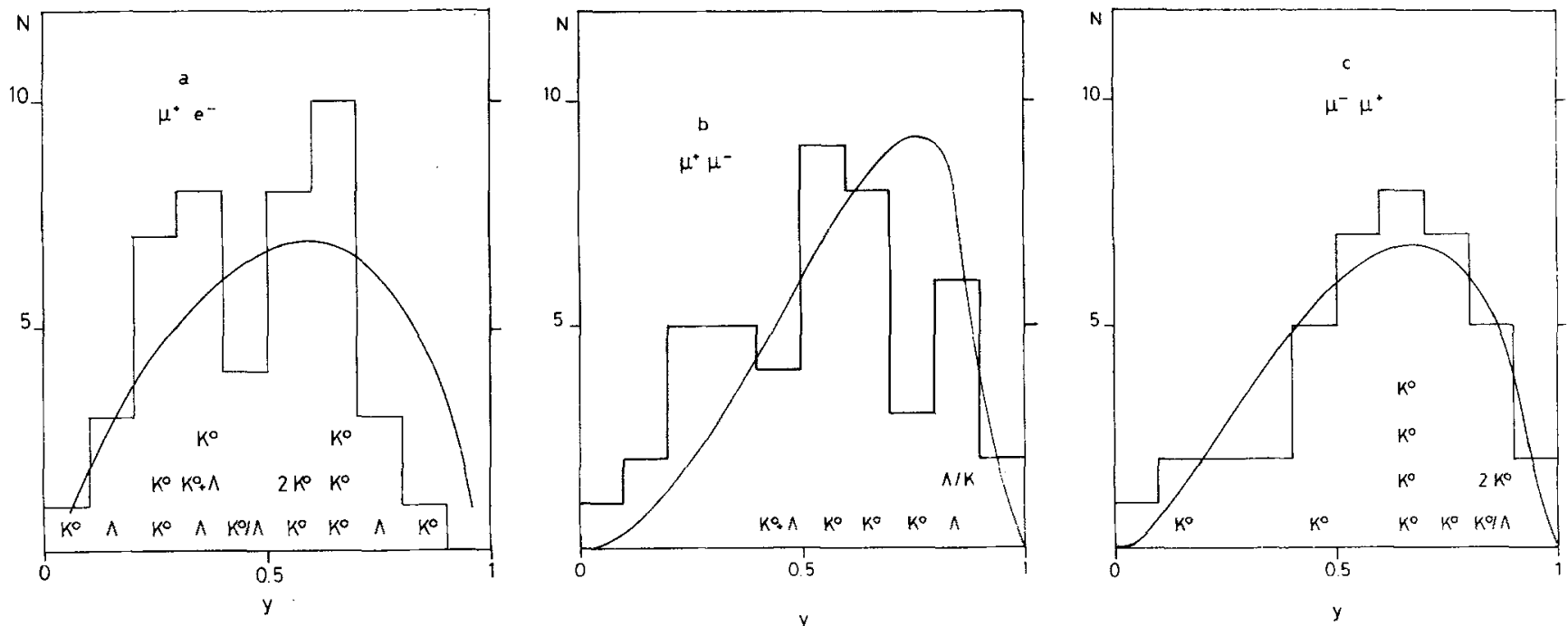

Fig. 5a-c. Distribution of the Bjorken $y$ variable, as for Fig. 2 

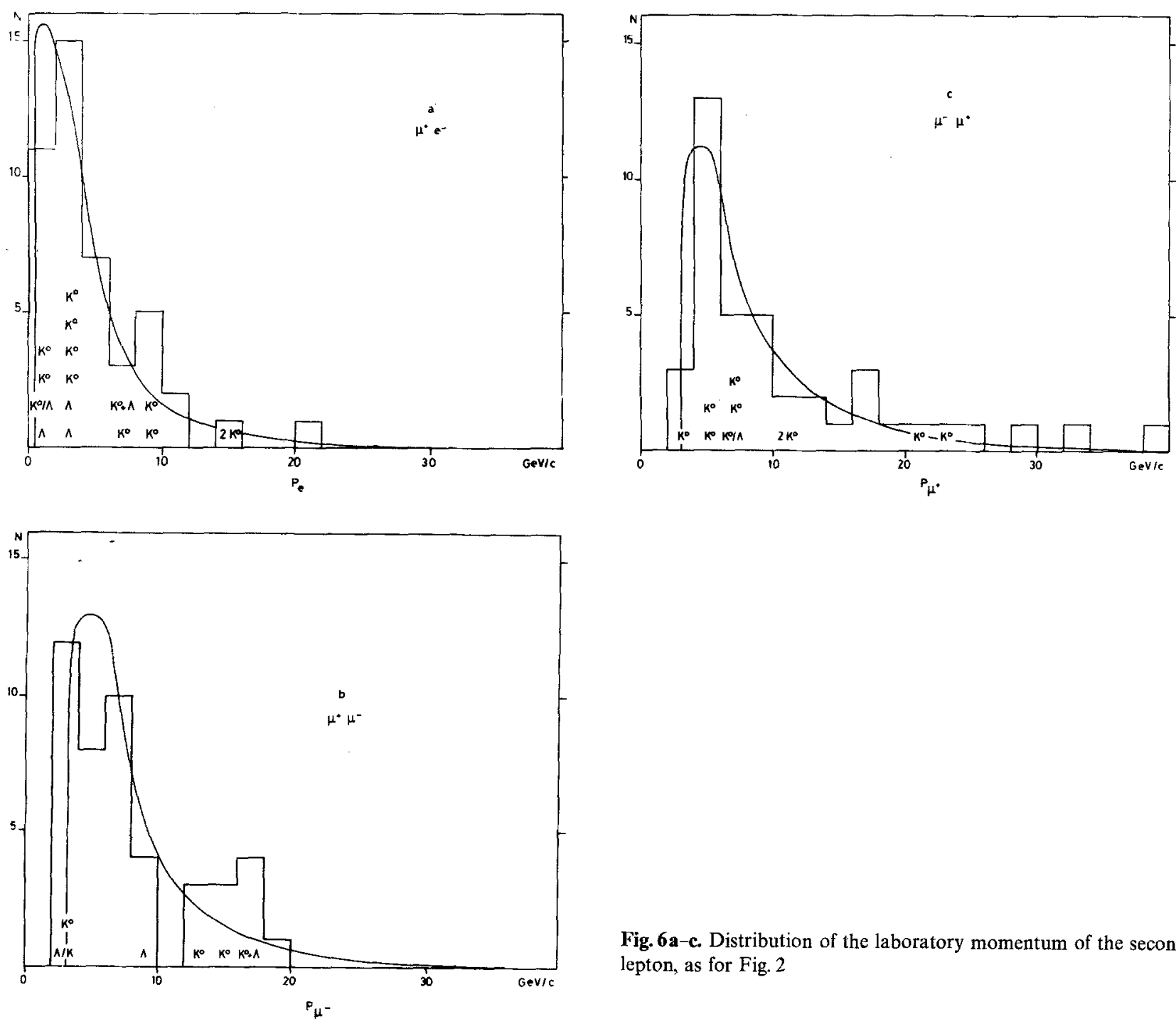

Fig. 6a-c. Distribution of the laboratory momentum of the second lepton, as for Fig. 2
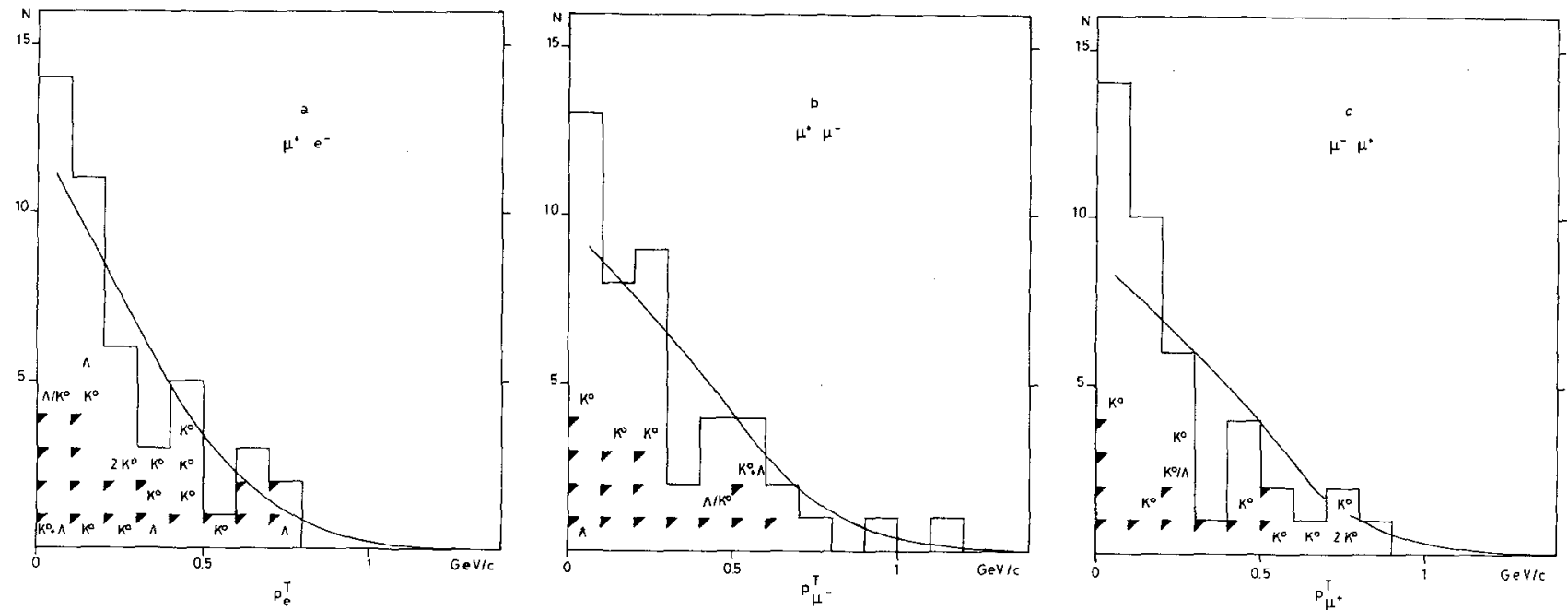

Fig. 7a-c. Distribution of the transverse momentum of the second lepton, with respect to the $\left(v, \mu_{1}\right)$ plane, as for Fig. 2 ; the events with $5<W<7 \mathrm{GeV}$ are labelled with a dark triangle 

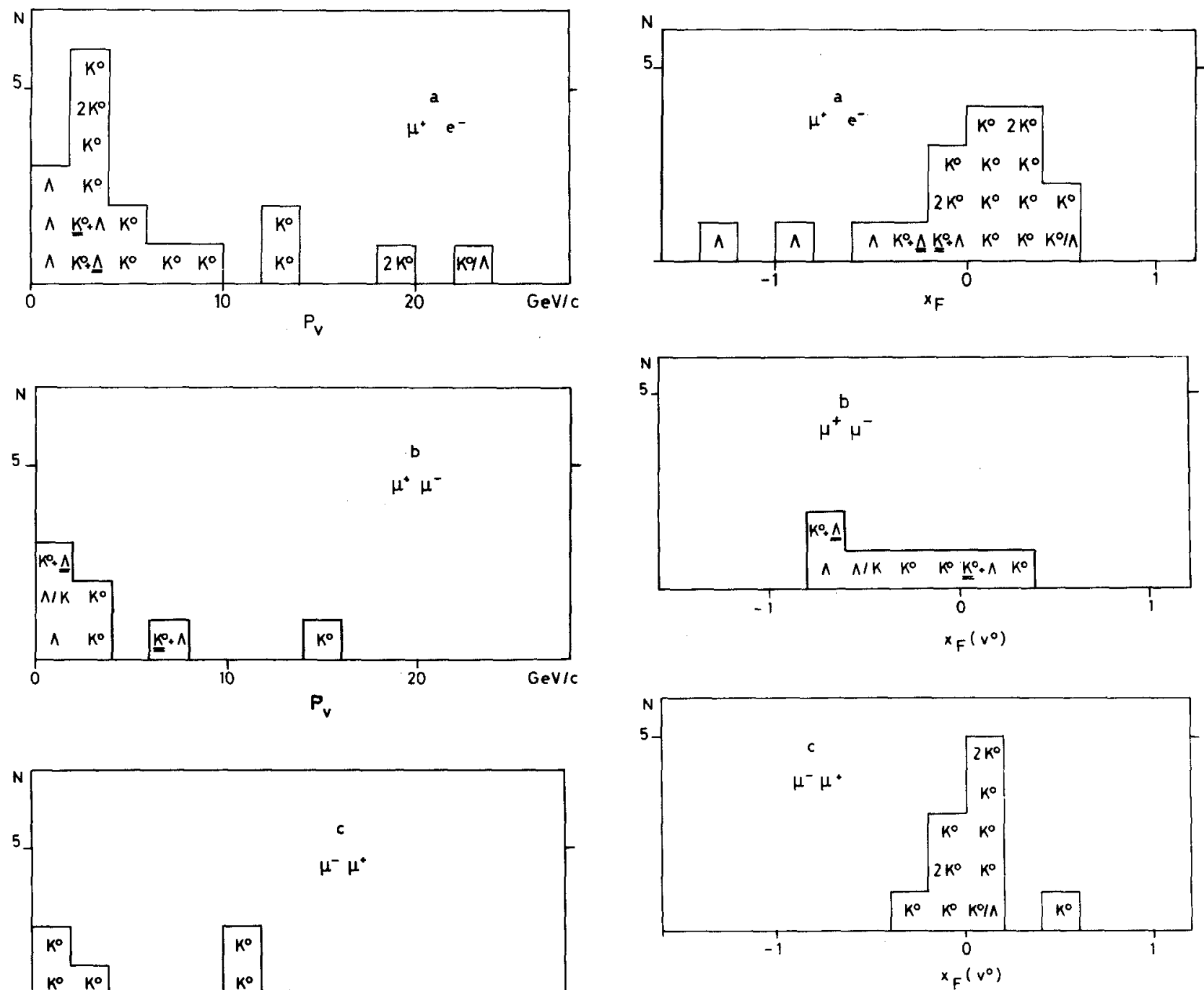

Fig. 9 a-c. Feynman $x$ distribution of the $V^{0}$, as for Fig. 8

Table 4. Measured same sign dilepton rates at $90 \%$ confidence level; $p_{\mu_{1}}>4 \mathrm{GeV} / \mathrm{c}, p_{\mu_{2}}>3 \mathrm{GeV} / \mathrm{c}, p_{e}>0.8 \mathrm{GeV} / \mathrm{c}$

Fig. 8a-c. Distribution of the laboratory momentum of the $V^{0}$ for: $\mathbf{a} \mu^{+} e^{-}$events ( $\bar{v}$ run); $\mathbf{b} \mu^{+} \mu^{-}$events ( $\bar{v}$ run); $\mathbf{c} \mu^{-} \mu^{+}$events ( $v$ run)

further $(1 \pm 1) e^{+}$is expected from $K^{+}$decays over the whole momentum range.

The $5 \mu^{+} \mu^{+}$candidates from the $\bar{v}$ run are compatible with the expected background of 7 events (see Table 1). One of these events contains a $A^{\circ}$ hyperon. The momentum distribution of the slower muon is also consistent with the soft distribution expected from $\pi$ and $K$ decays.

The $9 \mu^{-} \mu^{-}$candidates (including the trileptons here) from the $v$ run are also consistent with the expected 7 background events, as are the $4 \mu^{-} \mu^{-}$ candidates from the $\vec{v}$ run when 2 were expected. Of these 13 events, 4 have $p_{\mu_{2}}$ above $10 \mathrm{GeV} / \mathrm{c}$ where 2 events are expected. The $11 \mu^{-} \mu^{-}$events have no

$V^{0}$, but the 2 trileptons do include strange particles (see Sect. 7.5 and 7.6).

The limits on the like sign dileptons are given in Table 4. These limits assume that all events are compatible with background, and also that the slower muon, $\mu_{2}$, does not have a softer momentum spectrum than the $\mu_{2}$ in the opposite sign events. Any possible dilepton contribution from neutral current events is negligible, being about a factor $\mathrm{NC} / \mathrm{CC}$ times the charged current trilepton rate. 


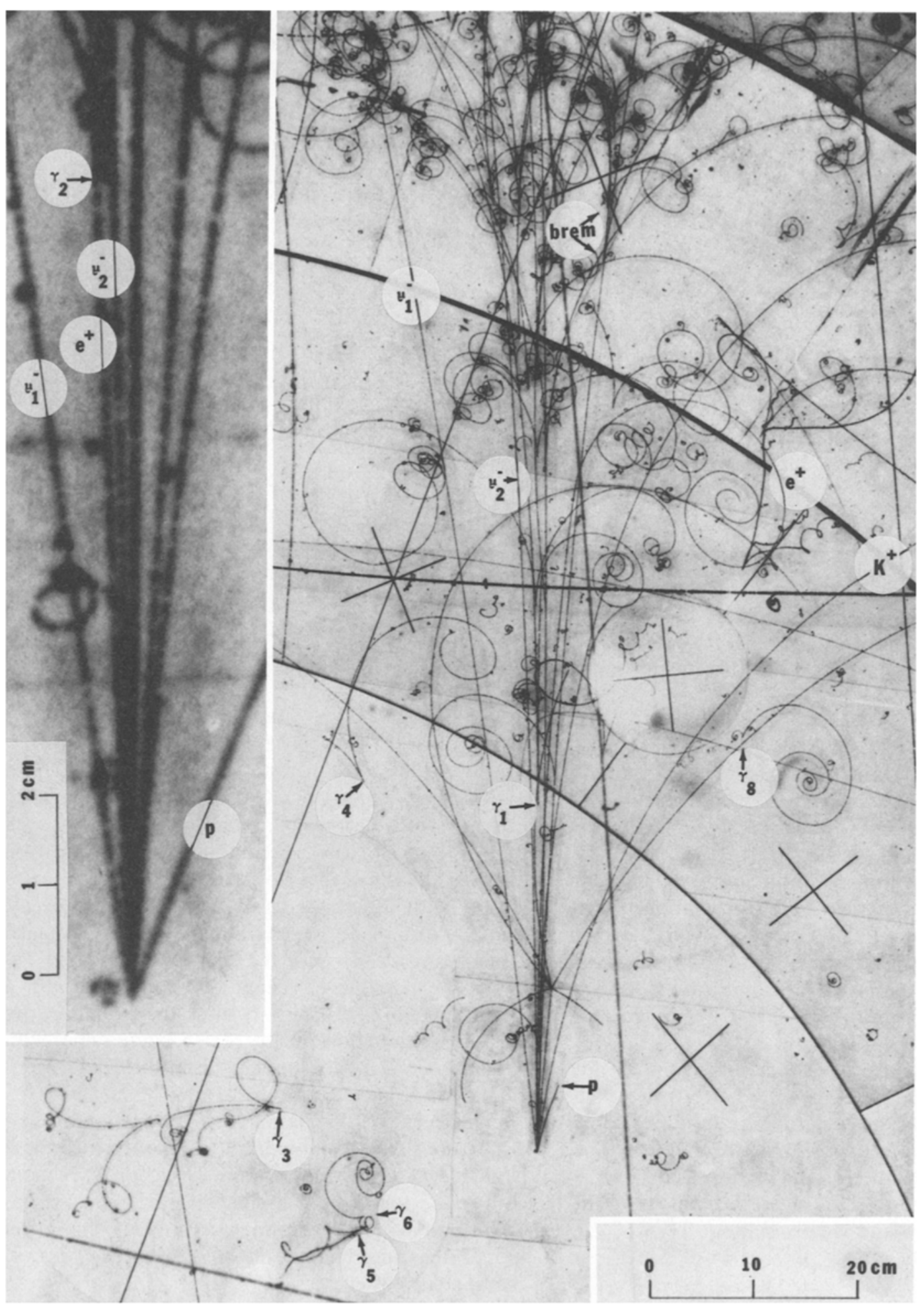

Fig. 10. The $\mu^{-} \mu^{-} e^{+}$trilepton event. The insert shows a detailed view of the vertex. (The $K^{+}$decay and $\gamma_{7}$ are not shown)

\subsection{The $\mu^{-} \mu^{-} \mu^{+}$Trilepton Event}

The $\mu^{-} \mu^{-} \mu^{+}$event has muons with momenta 84 , 4.9 and $6.2 \mathrm{GeV} / \mathrm{c}$. All have good association $\chi_{\mathrm{EMI}}^{2}$.
The two slower muons appear to be in the hadron shower, and have an invariant mass of (1.03 $\pm 0.02) \mathrm{GeV}$. There is also an $11 \mathrm{GeV} / \mathrm{c} K^{0}$ which may come from either the primary $v$ interaction, or 
Table 5. Kinematic variables of $\mu^{-} \mu^{-} e^{+}$trilepton and particle parameters: $E_{\mathrm{vis}}=109 \mathrm{GeV}, y_{\mathrm{vis}}=0.81, x_{\mathrm{vis}}=0.21, Q_{\mathrm{vis}}^{2}=34 \mathrm{GeV}^{2}, W_{\mathrm{vis}}^{2}$ $=132 \mathrm{GeV}^{2}$. Transverse momentum of $\mu_{1}^{-}$(to the beam) $=2.540 \pm 0.073 \mathrm{GeV} / \mathrm{c}$, missing transverse momentum $=1.8 \pm 0.3 \mathrm{GeV} / \mathrm{c}$

\begin{tabular}{|c|c|c|c|c|c|}
\hline & Particle & $\begin{array}{l}\text { Momentum } \\
(\mathrm{MeV} / \mathrm{c})\end{array}$ & $\begin{array}{l}\text { Dip angle } \\
\text { (mrad) }\end{array}$ & $\begin{array}{l}\text { Azimuthal angle } \\
\text { (mrad) }\end{array}$ & Identification \\
\hline 1 & $y$ & $>108,680$ & $42 \pm 2$ & $42 \pm 2$ & Beam \\
\hline 2 & $\mu_{1}^{-}$ & $20,813 \pm 459$ & $29 \pm 1$ & $163 \pm 1$ & EMI \\
\hline 3 & $\mu_{2}^{-}$ & $16,983 \pm 343$ & $17 \pm 1$ & $41 \pm 1$ & EMI \\
\hline 4 & $e^{+}$ & $5,344 \pm 1,076$ & $78 \pm 2$ & $67 \pm 5$ & Bremsstrahlung and curls up \\
\hline 5 & $I^{-}$ & $33,045 \pm 8,307$ & $24 \pm 2$ & $12 \pm 2$ & Interacts \\
\hline 6 & $L^{+}$ & $8,184 \pm 136$ & $43 \pm 1$ & $17 \pm 1$ & $\begin{array}{l}\text { Leaves chamber without identification: } \\
\text { but no EMI hits }\end{array}$ \\
\hline 7 & $I^{+}$ & $6,000 \pm 2,000$ & $42 \pm 7$ & $6,245 \pm 7$ & Interacts \\
\hline 8 & $\boldsymbol{K}^{+}$ & $1,046 \pm 51$ & $126 \pm 7$ & $6,210 \pm 6$ & $K^{+}$decay at rest \\
\hline 9 & $p$ & $377 \pm \quad 3$ & $-186 \pm 44$ & $6,069 \pm 26$ & $p$ stops \\
\hline 10 & $\gamma_{1}$ & $14,558 \pm 3,341$ & $54 \pm 2$ & $47 \pm 1$ & \\
\hline 11 & $\gamma_{2}$ & $2,281 \pm 744$ & $81 \pm 4$ & $39 \pm 2$ & \\
\hline 12 & $\gamma_{3}$ & $410 \pm \quad 78$ & $-844 \pm 3$ & $1,230 \pm 8$ & \\
\hline 13 & $\gamma_{4}$ & $246 \pm \quad 27$ & $213 \pm 3$ & $598 \pm 1$ & \\
\hline 14 & $\gamma_{5}$ & $98 \pm$ & $-1,457 \pm 3$ & $1,812 \pm 27$ & \\
\hline 15 & $\gamma_{6}$ & $38 \pm$ & $381 \pm 5$ & $2,033 \pm 4$ & \\
\hline 16 & $\gamma_{7}$ & $101 \pm$ & $500 \pm 1$ & $4,608 \pm 1$ & \\
\hline 17 & $\gamma_{8}$ & $93 \pm \quad 14$ & $-42 \pm 2$ & $5,840 \pm 1$ & \\
\hline
\end{tabular}

Invariant masses (in $\mathrm{MeV}$ ) $m\left(\gamma_{1} \gamma_{2}\right)=162 \pm 41 ; m\left(\gamma_{3} \gamma_{6}\right)=163 \pm 19 ; m\left(\gamma_{4} \gamma_{6}\right)=122 \pm 10 ; m\left(\gamma_{4} \gamma_{7}\right)=154 \pm 14 ; m\left(\gamma_{5} \gamma_{6}\right)=97 \pm 34 ; m\left(\gamma_{5} \gamma_{7}\right)=176$ $\pm 63 ; \quad m\left(\gamma_{5} \gamma_{8}\right)=137 \pm 48 ; \quad m\left(\gamma_{6} \gamma_{7}\right)=108 \pm 12 ; \quad m\left(\gamma_{6} \gamma_{8}\right)=111 \pm 11 ; \quad m\left(\gamma_{7} \gamma_{8}\right)=117 \pm 14 ; \quad m\left(\gamma_{4} \gamma_{8} \gamma_{5} \gamma_{7}\right)=465 \pm 84 ; \quad m\left(\mu_{2}^{-} K^{+}\right)=2,112 \pm 41 ;$ $m\left(e^{+} \gamma_{4} \gamma_{8} \gamma_{5} \gamma_{7}\right)=1,754 \pm 304$

Examples of products of possible semi-leptonic $D$ decays:

$m\left(e^{+}, I^{-}\right.$as $\left.K, \gamma_{1} \gamma_{2}\right)=1,699 \pm 208 ; m\left(\mu_{2}^{-}, L^{+}\right.$as $K, I^{+}$as $\left.\pi\right)=1,440 \pm 123$

from a secondary charged hadron interaction. The $\left(K^{0} \mu_{2}^{-}\right)$and $\left(K^{0} \mu^{+}\right)$invariant masses are $1.04 \mathrm{GeV}$ and $0.88 \mathrm{GeV}$. There are also 5 gammas, one interacting negative track and 2 tracks of unknown charge.

The high momentum $\mu^{-}$and lower momentum $\mu^{-} \mu^{+}$pair are compatible with expectations from other trilepton data [13]: consistent with normal charged current interactions with the additional production of lepton pairs by hadronic and radiative processes. One expects about $0.5 \mu l^{+} l^{-}$events in our data.

Since the muons are well identified they do not seem to be due to background. Nevertheless the most probable background interpretation of this event is as a genuine opposite sign dimuon with a background third "muon". The chance that there is one dimuon with a $\pi$ misidentified as a third "muon" in all our $v$ and $\bar{v}$ data is about 0.2 .

\subsection{The $\mu^{-} \mu^{-} e^{+}$Trilepton Event}

The muons in the $\mu^{-} \mu^{-} e^{+}$event have momenta 21 and $17 \mathrm{GeV} / \mathrm{c}$. These are both well identified, and have higher momenta than would be expected from decay background. The $(5 \pm 1) \mathrm{GeV} / \mathrm{c} e^{+}$can be followed clearly back to within $2 \mathrm{~cm}$ of the $v$ interaction and its only possible $e^{-}$partner is below
$2 \mathrm{MeV}$, (see Fig. 10). The second $\mu^{-}$and the $e^{+}$ appear to be in the hadron shower. The event also has a $1 \mathrm{GeV} / \mathrm{c}$ stopping $K^{+}$, and 6 low momentum gammas with various permutations consistent with three $\pi^{0}$; several two $\pi^{0}$ combinations may in turn have an invariant mass of about $500 \mathrm{MeV} / \mathrm{c}$, compatible with a fully reconstructed $K_{s}^{0} \rightarrow 2 \pi^{0}$ decay. The $K^{+}$cannot be paired with the leptons to make a mass compatible with the $D$ meson mass, see Table 5 . The possible $K_{s}^{0}$ cannot be combined with a $\mu^{-}$to make a $D$, but may be with the $e^{+}$. There are several combinations of particles compatible with semileptonic $D$ decay among the particles with momenta over $2 \mathrm{GeV} / \mathrm{c}$ : two are given.

This event requires a different production mechanism from previously reported trileptons, since these have had a leading muon and a $\mu^{+} \mu^{-}$or $e^{+} e^{--}$pair. However a four lepton event (with a $K_{s}^{0}$ decay) has been observed [14]. The single $\mu^{-} \mu^{-} e^{+}$event corresponds to a production rate of about $10^{-4}$ per $v_{\mu}$ charged current event.

The largest background is again from genuine opposite sign $\mu e$ events with an extra $\pi$ misidentified as a second " $\mu$ ". We expect about 0.2 such events in total, but only about 0.02 such events would be expected with a second " $\mu$ " over $10 \mathrm{GeV}$. There is a $1.5 \mathrm{MeV}$ electron at the vertex which we classify as a delta ray: the chance of seeing a delta ray over 
$1 \mathrm{MeV}$ within $1 \mathrm{~cm}$ of the vertex is about 0.3 . If classified as an $e^{-}$then the electron could be paired with the $e^{+}$. However less than 0.0001 dilepton events are expected to also have a Dalitz pair, or gamma within $1 \mathrm{~cm}$, with an $e^{+}$momentum over $3 \mathrm{GeV}$ and $e^{-}$less than $2 \mathrm{MeV}$.

\section{Discussion}

\section{1. $\mu^{+} e^{-} / \mu^{+} \mu^{-}$Comparison}

Although compatible with each other within statistics, some marginal differences could remain between the rates of $\mu^{+} e^{-}$and $\mu^{+} \mu^{-}$events and between the $V^{0}$ rates (see Table 3 ). It has been speculated [8] that such differences, if real, could arise from two body $F$ decays mediated by Higgs bosons [15], preferentially giving a muon as a second lepton, and no $V^{0}$. In this model one could expect the $p_{T}$ spectrum of the $\mu^{-}$to be harder than observed.

\subsection{Same Sign Dilepton Events}

The question of same sign dileptons has stimulated many discussions, because the high cross sections found in several experiments were not accounted for within the standard model: $c \bar{c}$ or beauty production [16].

Same sign dileptons have been observed in several counter experiments $[17$ to 22] and in a few bubble chamber experiments $\left(\mu^{+} e^{+}\right.$[23], $\mu^{-} e^{-}[41$, $24,25]$. The production rates differ widely and the experimental situation is unclear, mostly because of large uncertainties in subtracting the background in counter experiments, and because of the poor statistics in bubble chamber experiments. However our results and others $[3,26]$ and recent reanalyses of old data $[20,26]$ suggest the earlier rates may be too high.

For $\bar{v}$ interactions, the results presented in this paper $\left(\mu^{+} e^{+} / \mu^{+}<2 \times 10^{-4} ; \mu^{+} e^{+} / \mu^{+} e^{-}<6 \%\right)$ do not confirm the rate $4.8 \times 10^{-4}$ reported by Ammosov et al. [23] from $6,780 \bar{v}_{\mu}$ charged current events in similar experimental conditions. They saw $4 \mu^{+} e^{+}$events, $\left(p_{e^{+}}>0.3 \mathrm{GeV} / \mathrm{c}\right)$, with a total of $3 V^{0}$. One counter experiment [17] has reported a rate $\left(\mu^{+} \mu^{+} / \mu^{+} \mu^{-}\right)=(18 \pm 6 \pm 3) \%$, with $p_{\mu}>4 \mathrm{GeV} / \mathrm{c}$. This is both higher than our limit on the $\mu^{+} e^{+}$rate and also higher than our $\mu^{+} \mu^{+} / \mu^{+} \mu^{-}$limit of $12 \%$.

In $v$ interactions a Gargamelle experiment has reported a signal [4] of $(6 \pm 3)$ unweighted $\mu^{-} e^{-}$ events (with $p_{e^{-}}>0.5 \mathrm{GeV} / \mathrm{c}$ ) with no associated $V^{0}$ and all the electron momenta below $2 \mathrm{GeV} / \mathrm{c}$ (where the background contributions are greatest). The BNL-Columbia collaboration had previously re- ported $[24,25]$ the observation of $20 \mu^{-} e^{-}$ $\left(p_{e^{-}}>2 \mathrm{GeV} / \mathrm{c}\right)$, of which 11 were attributed to background, in a sample of some $100,000 \mathrm{CC}$ events. Recently [26] they have reported that there are no $\mu^{-} e^{-}$in a new sample of $51,000 \mathrm{CC}$ events (with $\left.p_{e^{-}}>4 \mathrm{GeV} / \mathrm{c}\right)$. Taking into account their electron detection efficiency, and correcting for the effect of the $p_{e^{-}}$cut using the model of Smith and Valenzuela [27], the new limit on $\mu^{-} e^{-}$is comparable to our limit on $\mu^{+} e^{+}$of $2 \times 10^{-4}$ for $p_{e}>0.8 \mathrm{GeV} / \mathrm{c}$. When comparing our results with those of counter experiments, the fact that the spectrum of the second lepton observed in those experiments seems to be softer in same sign than in opposite sign dileptons $[17,20]$ makes our $\bar{v}$ upper limit of $6 \%$ for $\mu^{+} e^{+} / \mu^{+} e^{-}$ harder to reconcile with some $\mu^{-} \mu^{-}$published rates $[17,19,22]$.

A model by Godbole and Roy [28], based on $c \bar{c}$ production in the hadron jet, could account for the like sign dimuons. The calculation by Smith and Valenzuela [27], extending this model to like sign $\mu e$ events, would predict about 6 prompt $\mu^{+} e^{+}$ events in our data: we see none. Such a model would, however, provide a natural explanation for a trilepton event such as the $\mu^{-} \mu^{-} e^{+}$from the $v$ run.

A model by Choban, which includes QCD and non-perturbative effects [29] appears to give like sign dimuon rates and kinematic distributions that are in agreement with earlier data. Choban's dilepton rates are for $E_{v}$ above $30 \mathrm{GeV}$ and our comparable $90 \%$ confidence level limit on $\left(\mu^{+} e^{+} / \mu^{+}\right)$becomes $4 \times 10^{-4}$ (for $p_{e^{+}}>0.8 \mathrm{GeV} / \mathrm{c}$ ), to be compared with Choban's theoretical estimate for $\mu^{+} \mu^{+} / \mu^{+}$ $=(3.3 \pm 0.2) \times 10^{-4}$ with $p_{\mu_{1}}>4 \mathrm{GeV} / \mathrm{c}$. However this theoretical $c \bar{c}$ rate seems higher than our upper limit since it would probably need to be enhanced by a factor of 3 to 4 to include the $e^{+}$with momenta between 0.8 and $4 \mathrm{GeV} / \mathrm{c}$.

A model by Cline [30] introduced leptonic decays of a new particle to account for some sign dileptons. Such decays have not contributed significantly to our data.

\section{Conclusions}

The results of this paper are based on a study of 192 dilepton events: these include the highest statistics to date on the production of dileptons by anti-neutrinos in bubble chambers. The kinematical distributions of the opposite sign dileptons are compatible with the expected production of charmed particles and their subsequent semi-leptonic decay, as in other experiments $[1,2]$.

The measured dilepton rates and associated $V^{0}$ yields are shown in Table 3 . This experiment is un- 
able to conclude whether remaining small differences between the $\mu^{+} \mu^{-}$and $\mu^{+} e^{-}$rates indicate any additional production mechanisms.

The present experiment shows no evidence for prompt like sign dilepton production. Our limit on $\mu^{+} e^{+} / \mu^{+}$imposes important restrictions on $c \bar{c}$ production models since there is little background to the $e^{+}$whereas the $\mu^{-} e^{-}$data include Compton scattering background and decay backgrounds are large in $\mu \mu$. Our lack of prompt same sign dileptons is hardly compatible with rates observed in-some bubble chamber and counter experiments which have been considered as important support for the enhanced $c \bar{c}$ production models of Godbole and Roy (extended by Smith and Valenzuela), and of Choban, and also for the different model of Cline.

The $\mu^{-} \mu^{-} e^{+}$trilepton event requires a different production mechanism from previously reported trileptons, but could by explained by $c \bar{c}$ production.

Acknowledgements. We would like to thank the BEBC and EMI crews for the excellent data, and our scanning and measuring teams for their careful work.

\section{References}

1. See for example H. Abramowicz et al.: Z. Phys. C - Particles and Fields 15, 19 (1982)

2. See for example N. Armenise et al.: Phys. Lett. 86 B, 115 (1979), and [3-5]

3. H.C. Ballagh et al.: Phys. Rev. D 24, 7 (1981)

4. A. Haatuft et al:: Nucl. Phys B 222, 365 (1983)

5. N.J. Baker et al.: Phys. Rev. D 32, 531 (1985)
6. P. Marage et al.: Z. Phys. C - Particles and Fields 21, 307 (1984)

7. R. Beuselinck et al.: Nucl. Instrum. Methods 154, 445 (1978)

8. G. Gerbier: Thesis presented at L'Université d'Orsay (1984)

9. R.D. Field, R.P. Feyman: Phys. Rev. D 15, 2590 (1977)

10. N. Armenise et al.: Phys. Lett. 94 B, 527 (1980)

11. B.W. Lee, R.P. Schrock: Phys. Rev. D 13, 2359 (1976)

12. K. Kleinknecht, B. Renk: Phys. Lett. 130 B, 459 (1983), Table 4

13. T. Hansl et al.: Phys. Lett. 77 B, 114 (1978)

14. R.J. Loveless et al.: Phys. Lett. 78 B, 505 (1978)

15. V. Barger et al.: Phys. Lett. 116 B, 357 (1982)

16. V. Barger: Proceedings of the XXI International Conference in High Energy Physics, Paris 1982, pC3-32; F. Halzen: ibid, pC3-381

17. M. Jonker et al.: Phys. Lett. 107 B, 241 (1981)

18. J.G.H. de Groot et al.: Phys. Lett. 86 B, 103 (1979)

19. T. Trinko et al.: Phys. Rev. D 23, 1889 (1981)

20. K. Nishikawa et al.: Phys. Rev. Lett. 46, 1555 (1981) and erratum K. Nishikawa et al.: Phys. Rev. Lett. 54, 1336 (1985)

21. J. Knobloch: Proceedings of the 1981 International Conference on Neutrino Physics and Astrophysics, Vol. I, p. 421. Hawaii 1981

22. K. Kleinknecht: Proceedings of the International Conference, Neutrino 1982, Vol. I, p. 115. Budapest 1982

23. V.V. Ammosov et al.: Phys. Lett. 106 B, 151 (1981)

24. M.J. Murtagh: Proceedings of the 1979 International Symposium on Lepton and Photon Interactions at High Energy, p. 277. Fermilab 1979

25. C. Baltay: Proceedings of 1979 JINR-CERN School of Physics, p. 64. Dobogoko 1979, Budapest: Hungarian Academy of Science 1980

26. A. Schaffer: Report at the American Physical Society, Santa $\mathrm{Fe}(1984)$

27. J. Smith, G. Valenzuela: Phys, Rev. D 28, 1071 (1983)

28. R.M. Godbole, D.P. Roy: Phys. Rev. Lett. 48, 1711 (1982); Z. Phys. C - Particles and Fields 22, 39 (1984)

29. E.A. Choban: Z. Phys. C - Particles and Fields 25, 269 (1984)

30. D.B. Cline: Lett. Nuovo Cimento 40, 377 (1984) 